\title{
Mycobacterium tuberculosis: Active disease and latent infection in a renal transplant cohort
}

\author{
Nastaran Rafiei ${ }^{1}$, Jackie Williams ${ }^{1}$, William R. Mulley ${ }^{2,3}$, James M Trauer ${ }^{4}$, Grant A Jenkin ${ }^{1}$, \\ Benjamin A Rogers ${ }^{1,3}$
}

1. Monash Infectious Diseases, Monash Health, Melbourne Australia;

2. Department of Nephrology, Monash Medical Centre, Clayton, Vic, Australia;

3. Centre for Inflammatory Diseases, Department of Medicine, Monash University, Clayton, Vic, Australia

4. School of Public Health and Preventive Medicine, Monash University, Victoria, Australia

Corresponding author: Benjamin A Rogers

Address: 246 Clayton Rd, Clayton VIC 3168

Email: ben.rogers@monash.edu

Telephone: +61395946666

Running title: Active and latent TB in renal transplant

This is the author manuscript accepted for publication and has undergone full peer review but has not been through the copyediting, typesetting, pagination and proofreading process, which may lead to differences between this version and the Version of Record. Please cite this article as doi: $10.1111 /$ nep.13386

This article is protected by copyright. All rights reserved. 


\begin{abstract}

\section{Background}

Tuberculosis (TB) is a serious opportunistic infection in renal transplant recipients associated with high mortality. Screening and treatment of latent Mycobacterium tuberculosis infection (LTBI) offers an opportunity to prevent subsequent active disease.
\end{abstract}

\title{
Methods
}

We retrospectively reviewed the records of all adult patients who underwent renal transplantation at our centre from 2005 to 2014 to assess current screening practices, the risks for and burden of active TB.

\section{Results}

A total of 660 individuals underwent renal transplantation during this period, totalling 3647 person years of follow up. Three patients were diagnosed with active TB after renal transplant, resulting in an incidence of 82 per 100,000 person-years. Of 656 transplant recipients, $102(15.5 \%)$ were born in high TB incidence countries and $89(13.5 \%)$ had an interferon gamma release assay (IGRA) at any point. Individuals born in high TB risk countries had a much higher incidence of active TB (530 per 100,000 person-years). Ten individuals had positive IGRA tests, of whom two were treated for active TB, two received chemoprophylaxis and six were not treated.

\section{Conclusions}

In the absence of formal guidelines, IGRA-based screening for LTBI was infrequently performed. Our data suggests that screening and treatment of renal transplant recipients born in high incidence countries is an important preventive measure.

This article is protected by copyright. All rights reserved. 
Keywords: adults, chemoprevention, kidney transplantation, opportunistic infections, tuberculosis.

\section{Introduction}

Mycobacterium tuberculosis $(\mathrm{Mtb})$ is one of the most common infections globally, with around one quarter of the world's population infected. ${ }^{1}$ The vast majority have latent infection and will remain asymptomatic throughout their lifetime. In low-incidence settings, active (symptomatic) tuberculosis (TB) disease is most commonly due to reactivation of latent $M t b$ infection (LTBI), and immune suppression increases the risk of reactivation. ${ }^{2-4}$

Similarly, in the transplant population, TB most commonly arises due to reactivation in the setting of immunosuppressive medications. ${ }^{5}$ Less commonly it may arise from primary infection or donor derived infection. ${ }^{6}$ Solid organ transplant recipients are at increased risk of TB, with rates 20 to 74 times background rates and this can reach $15 \%$ in high-incidence countries. ${ }^{7}$ In transplant recipients,

TB may present atypically and be associated with significant diagnostic delays. ${ }^{5,8}$ In addition to direct morbidity and mortality, TB can cause graft dysfunction and may constitute a public health risk if involving the lungs, while treatment is complicated by drug-drug interactions with immunosuppressive medications. ${ }^{5}$ The ability to screen for LTBI offers an opportunity to implement chemoprophylaxis and diminish the risk of reactivation of TB. Isoniazid preventive therapy (IPT) given for 6 to 12 months is the most commonly used treatment regimen and is $60-70 \%$ effective at preventing active disease. ${ }^{9-12}$

Renal transplantation has been offered at our centre since 1993, with the number of transplants increasing over this time. The World Health Organization, as well as many national transplant committees, have formulated guidelines for the management of LTBI in solid organ transplant 
recipients, but at present, LTBI screening is not a part of Australian renal transplant guidelines. ${ }^{9-11,13-}$

${ }^{15}$ Furthermore, little has been published about TB in renal transplant cohorts from low TB incidence countries. Therefore there has been no protocol or uniform approach to TB screening for renal transplant recipients at our institution. One explanation for the absence of direction on this issue is the very low rate of TB in Australia. According to the most recent notification data, Australia had an incidence of active TB of 5.5 per 100,000, which is one of the lowest rates in the world. The vast majority of cases occur in those born overseas, with $88 \%$ of TB notifications occurring in overseasborn individuals in 2013 .

Our aim was threefold: first, to determine the incidence of active TB in our cohort, second to investigate the risk factors for active TB and third, to understand current screening practices. The ultimate goal was to use our findings to inform development of local and national guidelines.

\section{Methods}

\section{Study population}

Our study population was adults (age e 16) who underwent renal transplantation at our institution from 2005 to 2014 inclusive, including those who had simultaneous pancreas-kidney (SPK) transplants.

Patients were initially identified from a prospectively collected database managed by the renal transplant service. Data were cross-referenced and further details sought from the Australian and New Zealand dialysis and transplant registry (ANZDATA). Background demographic and patient comorbidity data were extracted. Comorbidity information on ANZDATA is based on the treating clinician recording whether the patient has coronary artery disease, chronic lung disease, cerebrovascular disease, peripheral vascular disease or diabetes according to their clinical opinion. 


\section{Country of birth}

We collected country of birth $(\mathrm{COB})$ information for individuals transplanted in this 10 year period and classified them into high and low-risk countries. High-risk countries were defined as those with a TB incidence of 40 cases per 100, 000 persons per year or greater according to World Health Organization estimates of TB incidence in 2015. ${ }^{16}$ All other countries were categorised as lowincidence countries.

\section{IGRA testing and active tuberculosis}

IGRA testing and active TB disease were identified by cross-searching our health service's microbiology laboratory information system and the renal transplant database. IGRA testing was performed using the QuantiFERON-TB Gold assay (Cellestis, Melbourne Australia). Tests were identified regardless of whether they were performed before or after transplantation. A positive IGRA response was defined as a TB antigen tube minus nil tube response of e $0.35 \mathrm{IU} / \mathrm{ml}$ and $<25 \%$ of the nil tube with a mitogen response of $>0.5 \mathrm{IU} / \mathrm{mL}$, in accordance with the manufacturer's instructions. Any test which failed to elicit a mitogen response $(<0.5 \mathrm{IU} / \mathrm{ml})$ was classified as indeterminate. For those individuals who had a positive IGRA, the medical records were reviewed to determine their clinical management.

Individuals who were transplanted more than once in this period were classified only in the year of first transplantation. For those individuals who had more than one IGRA with discordant results, the non-indeterminate result is recorded. If a person returned both a positive and negative result, the result was considered positive. Active TB was defined by a clinical specimen which grew Mtb complex or was positive for $M t b$ DNA via nucleic acid amplification testing (NAAT). 
Total person-years of follow up was determined by calculating the time from transplantation to the study end date or time of death.

\section{Statistical analysis}

Univariate analyses were performed using chi-square or Fisher's exact test with a p-value of $<0.05$ considered significant. The number needed to prevent was calculated as the expected number of individuals from high-incidence countries with a positive or indeterminate IGRA who would have been eligible for IPT, divided by the number of cases of active TB observed and the effectiveness of the preventive treatment. The study was exempt from submission to the human research ethics committee as a quality improvement activity.

\section{Results}

Over the 10 year period from 2005 to 2014, a total of 671 renal transplants (including 111 SPK transplants and 11 repeat transplants) were performed in 660 individuals, totalling 3647 person-years of follow up.

Patient characteristics are presented in Table 1. The median age at transplantation was 50 (interquartile range $39-59$ ) and $62.7 \%$ of recipients were male. The most common primary cause of renal disease was glomerulonephritis (37.4\%), followed by diabetes $(29.7 \%)$.

\section{Birth in a high TB-incidence country}

Country of birth was recorded for 656 of 660 (99.4\%) patients, of which $102(15.5 \%)$ were born in high-risk countries. The five most common high-incidence countries were Sri Lanka (21), India (16), Vietnam (13), Cambodia (9) and Fiji (8). The majority of patients were Australian born (415; 63.3\%). The next most common countries of birth were the United Kingdom $(n=32 ; 4.9 \%)$, Sri Lanka $(n=21$; 
$3.2 \%)$ and India $(n=16 ; 2.4 \%)$. There was no marked change in the frequency of birth in highincidence countries over the study period (Figure 1).

\section{IGRA testing}

One hundred and twenty-three IGRA tests were carried out on 89 individuals (13.5\%) of the cohort (Table 1). Individuals who underwent IGRA testing were more likely to have been born in countries with high TB incidence ( $42.7 \%$ vs $11.3 \%$; $\mathrm{p}=0.001$ Fisher's exact). Additionally, they were less likely to have diabetes $(18.0 \%$ vs $31.5 \% ; \mathrm{p}=0.016)$ or have received a SPK transplant $(6.7 \%$ vs $18.4 \%$; $\mathrm{p}=0.015)$. The characteristics of the patients who had IGRA testing correlated with those of the whole cohort born in high TB incidence countries.

The screening and treatment cascade is shown in Figure 2. Of the 28 individuals who had IGRA testing performed prior to transplantation, $13(46.4 \%)$ were born in high TB incidence countries and 3 had positive results. The majority of IGRA tests $(70.8 \%)$ were performed post-transplantation. Twenty-two individuals had more than one test performed, and ten (45.5\%) had discordant results. All except one of these were negative in one test and indeterminate in another. For one individual who had three IGRA tests performed, results were positive in two instances and indeterminate in one. Indeterminate results were returned in six of 28 (21.4\%) individuals tested pre-transplantation and 9 of $61(14.8 \%)$ individuals tested post-transplantation.

Of the ten patients who returned positive tests, seven were born in high TB incidence countries. The most common indication for testing was potential active TB and this was confirmed in two patients who were subsequently treated. A further two patients received IPT for latent disease. Six patients had 
positive IGRA results which were not acted upon; none were documented to have contraindications to IPT.

Thirty-eight individuals born in high TB incidence countries had IGRA testing $(38 / 102 ; 37.3 \%)$ at any time and three received IPT.

\section{Active tuberculosis disease}

Three patients, none of whom were screened pre-transplant with IGRA, developed culture-positive during the study period. The clinical characteristics of these patients are shown in Table 2. TB was diagnosed from ten months to 15 years post-transplantation. All three patients had significant adverse effects throughout treatment and 1 patient died whilst on the continuation phase of therapy. The death was assessed as not attributable to TB by the treating clinicians. For the entire cohort of 660 transplant recipients with a total of 3647 person-years of follow up, the rate of active TB was 82 cases per 100,000 person-years. Active TB occurred exclusively in individuals born in high incidence countries, and in this subgroup of 102 individuals with 566.5 person-years of follow up, the rate was substantially higher at 530 per 100,000 person-years.

Among the 38 individuals from high TB incidence countries who underwent IGRA testing, 29\% (11 patients) tested positive or indeterminate. Based on this proportion, we would expect approximately 30 individuals ( $102 \times 29 \%$ ) would have tested positive or indeterminate over the ten year study period had IGRA testing been carried out systematically. Assuming an effectiveness of IPT of $60-70 \%$, we would have treated 30 individuals to prevent two of the three cases of active TB described. This gives a number needed to prevent of 15 per active TB case. 


\section{Discussion}

We found that despite approximately $16 \%$ of our renal transplant population being at risk of TB according to their countries of birth, in the absence of a formal protocol, screening for TB with IGRA was inconsistently performed. Only 28 patients (4.2\%) were tested prior to transplantation and $37.3 \%$ of those from high TB incidence countries had IGRA testing at any stage. Most IGRA tests $(70.8 \%)$ were performed post-transplant, suggesting that they were performed for the purposes of investigation into active TB, rather than screening for LTBI. Once active disease had been excluded, few patients were treated for LTBI as clinicians did not appear to consider the implications of a positive IGRA result. No patient was documented to have a contraindication to IPT.

In our cohort of 660 patients, we found three cases of active TB over 3647 person years of follow up, with cases occurring exclusively in individuals born in high-incidence countries. The incidence in this subgroup (530 per 100,000 person-years) is equivalent to the rate in some of the highest incidence countries in the world.

Although it is generally agreed that there is a need to assess for and treat LTBI in transplant recipients, consensus has not been reached on the optimal management approach. One of the major issues has been the absence of a reliable screening test. Published guidelines provide differing recommendations but most suggest a combination of risk factor assessment, chest $\mathrm{x}$-ray, IGRA and/or tuberculin skin test (TST). ${ }^{9-11}$ IGRA testing has the benefits of greater specificity and ease of administration over the traditionally used TST. However, false negative results can still occur, particularly in those with deficiencies in cell-mediated immunity. ${ }^{17,18}$ Of note, one of our patients had a negative IGRA just prior to the diagnosis of active $\mathrm{TB}$, highlighting the fact that a negative IGRA test does not exclude a diagnosis of TB. 
A second important drawback of IGRAs is indeterminate results which occur because of an inadequate mitogen response in the setting of immune suppression. Indeterminate rates were $21.4 \%$ pre-transplantation in our cohort. Others have reported rates of indeterminate results of $1-20 \%$, with higher rates seen when testing is performed after transplantation. Notably, the rates of indeterminate rates were not higher post-transplantation in our patients.

Furthermore, although IPT is well recognised to be beneficial in preventing TB in immunocompetent hosts, only a handful of prospective randomised studies have been performed evaluating the efficacy of IPT in renal transplant patients. ${ }^{19,20}$ A meta-analysis of the four randomised trials performed in TB endemic settings (India and Pakistan) showed a protective benefit of 12 months of IPT in renal transplant patients with a relative risk of 0.31 . Importantly, there was no significant difference in rates of hepatitis between those who received IPT and those who did not. ${ }^{21}$ These studies have shown much higher rates of TB (up to $33 \%$ in the placebo arm) and viral hepatitis and variable screening protocols for LTBI. ${ }^{22-25}$ For these reasons, direct extrapolation of this data to our setting is not possible. A South Korean study evaluating 9 months of IPT versus placebo for renal transplant patients after screening for LTBI showed a non-significant trend towards reduction in TB $(2 \% \mathrm{vs} 0 \%$; $\mathrm{p}=0.09){ }^{26}$

Limitations of our study include that we used only those IGRA and pathology tests performed by our hospital-based pathology provider. Screening tests performed at other pathology providers may therefore have been missed. However, as all tests pre-transplantation are performed in this centre, it is unlikely that a high number of tests were missed. It is also possible that patients who moved out of our catchment area subsequently developed active TB, which was not captured in our study although 
this occurs infrequently as post-transplant care is provided by a limited number of publically funded hospitals in our region. Another limitation is that we did not consider the use of TSTs or chest X-rays as screening tests. Although TST has been available at our centre until recently, our standard screening test during the study period was IGRA. As we performed univariate analyses, there is the potential of multiple comparisons leading to inappropriate attribution of significance. Only a small number of active TB cases and a relatively low number of IGRAs were performed in our cohort, which formed the basis of our number needed to prevent estimate. However, supporting our data, Kim et al found a similar rate of active TB (490 per 100,000 person years) in their study of active TB in kidney transplant recipients in South Korea. ${ }^{26}$

In conclusion, we show up to $16 \%$ of our renal transplant patients may be at risk of TB according to their birth countries. Without a formal protocol, screening for LTBI with IGRA was infrequently performed.

We found a high rate of active TB of 530 per 100,000 person-years in those patients born in high TB incidence countries. No patients from low-incidence countries developed active TB, suggesting that systematically screening this cohort would be unnecessary. Although the absolute number of individuals who developed active TB following transplantation was low, all three had significantly difficult treatment courses. All 3 had disease involving the lungs and were therefore at risk of transmitting infection in the community. Our study shows that a relatively small number of individuals would need to undergo IPT prophylaxis if screening with IGRA was systemically applied and targeted to those at highest risk. However given the issues with IGRA testing in immunocompromised populations, this test by itself may not be an adequate form of screening. ${ }^{27-29}$ Rather it may be more useful as one part of a multi-faceted risk assessment. A reasonable approach to 
screening for renal transplant candidates would be initial risk assessment based on country of birth, and further screening with chest x-ray and IGRA for those from high-TB incidence countries.

This article is protected by copyright. All rights reserved. 
Acknowledgements: None

Conflict of interest: None to declare.

Funding: No funding was received

This article is protected by copyright. All rights reserved. 


\section{References:}

1. Houben RM, Dodd PJ. The Global Burden of Latent Tuberculosis Infection: A Re-estimation Using Mathematical Modelling. 2016;13(10):e1002152.

2. Pareek M, Watson JP, Ormerod LP, Kon OM, Woltmann G, White PJ, et al. Screening of immigrants in the UK for imported latent tuberculosis: a multicentre cohort study and costeffectiveness analysis. The Lancet Infectious Diseases. 2011;11(6):435-44.

3. Toms C, Stapledon R, Waring J, Douglas P. Tuberculosis notifications in Australia, 2012 and 2013. Commun Dis Intell Q Rep. 2015;39(2):E217-35.

4. Horsburgh CRJ, Rubin EJ. Latent Tuberculosis Infection in the United States. New England Journal of Medicine. 2011;364(15):1441-8.

5. Singh N, Paterson DL. Mycobacterium tuberculosis infection in solid-organ transplant recipients: impact and implications for management. Clinical infectious diseases : an official publication of the Infectious Diseases Society of America. 1998;27(5):1266-77.

6. Mourad G, Soulillou JP, Chong G, Pouliquen M, Hourmant M, Mion C. Transmission of Mycobacterium tuberculosis with renal allografts. Nephron. 1985;41(1):82-5.

7. Munoz L, Santin M. Prevention and Management of Tuberculosis in Transplant Recipients: From Guidelines to Clinical Practice. Transplantation. 2016;100(9):1840-52.

8. Subramanian AK, Morris MI. Mycobacterium tuberculosis infections in solid organ transplantation. American journal of transplantation : official journal of the American Society of Transplantation and the American Society of Transplant Surgeons. 2013;13 Suppl 4:68-76.

9. Bumbacea D, Arend SM, Eyuboglu F, Fishman JA, Goletti D, Ison MG, et al. The risk of tuberculosis in transplant candidates and recipients: a TBNET consensus statement. The European respiratory journal. 2012;40(4):990-1013.

10. Meije Y, Piersimoni C, Torre-Cisneros J, Dilektasli AG, Aguado JM. Mycobacterial infections in solid organ transplant recipients. Clinical microbiology and infection : the official publication of the European Society of Clinical Microbiology and Infectious Diseases. 2014;20 Suppl 7:89-101.

11. Aguado JM, Torre-Cisneros J, Fortun J, Benito N, Meije Y, Doblas A, et al. Tuberculosis in solid-organ transplant recipients: consensus statement of the group for the study of infection in transplant recipients (GESITRA) of the Spanish Society of Infectious Diseases and Clinical Microbiology. Clinical infectious diseases : an official publication of the Infectious Diseases Society of America. 2009;48(9):1276-84.

12. Jasmer RM, Nahid P, Hopewell PC. Clinical practice. Latent tuberculosis infection. The New England journal of medicine. 2002;347(23):1860.

13. Getahun H, Matteelli A, Abubakar I, Aziz MA, Baddeley A, Barreira D, et al. Management of latent Mycobacterium tuberculosis infection: WHO guidelines for low tuberculosis burden countries. The European respiratory journal. 2015;46(6):1563-76.

14. Campbell S, Pilmore H, Gracey D, Mulley W, Russell C, McTaggart S. KHA-CARI guideline: recipient assessment for transplantation. Nephrology (Carlton, Vic). 2013;18(6):455-62.

15. Milburn H, Ashman N, Davies P, Doffman S, Drobniewski F, Khoo S, et al. Guidelines for the prevention and management of Mycobacterium tuberculosis infection and disease in adult patients with chronic kidney disease. Thorax. 2010;65(6):557-70.

16. WHO. Global tuberculosis report 2016.

17. Pai M, Zwerling A, Menzies D. Systematic review: T-cell-based assays for the diagnosis of latent tuberculosis infection: an update. Annals of internal medicine. 2008;149(3):177-84. 
18. Pai M, Denkinger CM, Kik SV, Rangaka MX, Zwerling A, Oxlade O, et al. Gamma interferon release assays for detection of Mycobacterium tuberculosis infection. Clinical microbiology reviews. 2014;27(1):3-20.

19. Efficacy of various durations of isoniazid preventive therapy for tuberculosis: five years of follow-up in the IUAT trial. International Union Against Tuberculosis Committee on Prophylaxis. Bulletin of the World Health Organization. 1982;60(4):555-64.

20. Smieja MJ, Marchetti CA, Cook DJ, Smaill FM. Isoniazid for preventing tuberculosis in nonHIV infected persons. The Cochrane database of systematic reviews. 2000(2):Cd001363.

21. Currie AC, Knight SR, Morris PJ. Tuberculosis in renal transplant recipients: the evidence for prophylaxis. Transplantation. 2010;90(7):695-704.

22. Naqvi R, Akhtar S, Noor H, Saeed T, Bhatti S, Sheikh R, et al. Efficacy of isoniazid prophylaxis in renal allograft recipients. Transplantation proceedings. 2006;38(7):2057-8.

23. John GT, Thomas PP, Thomas M, Jeyaseelan L, Jacob CK, Shastry JC. A double-blind randomized controlled trial of primary isoniazid prophylaxis in dialysis and transplant patients. Transplantation. 1994;57(11):1683-4.

24. Agarwal SK, Gupta S, Dash SC, Bhowmik D, Tiwari SC. Prospective randomised trial of isoniazid prophylaxis in renal transplant recipient. International urology and nephrology. 2004;36(3):425-31.

25. Vikrant S, Agarwal SK, Gupta S, Bhowmik D, Tiwari SC, Dash SC, et al. Prospective randomized control trial of isoniazid chemoprophylaxis during renal replacement therapy. Transplant infectious disease : an official journal of the Transplantation Society. 2005;7(3-4):99-108.

26. Kim SH, Lee SO, Park IA, Kim SM, Park SJ, Yun SC, et al. Isoniazid treatment to prevent TB in kidney and pancreas transplant recipients based on an interferon-gamma-releasing assay: an exploratory randomized controlled trial. The Journal of antimicrobial chemotherapy.

2015;70(5):1567-72.

27. Hadaya K, Bridevaux PO, Roux-Lombard P, Delort A, Saudan P, Martin PY, et al. Contribution of interferon-gamma release assays (IGRAs) to the diagnosis of latent tuberculosis infection after renal transplantation. Transplantation. 2013;95(12):1485-90.

28. Ishikawa S, Igari H, Akutsu N, Tsuyuzaki M, Aoyama H, Hasegawa M, et al. Comparison of interferon-gamma release assays, QuantiFERON TB-GIT and T-Spot.TB, in renal transplantation. Journal of infection and chemotherapy : official journal of the Japan Society of Chemotherapy. 2017;23(7):468-73

29. Edathodu J, Varghese B, Alrajhi AA, Shoukri M, Nazmi A, Elgamal H, et al. Diagnostic potential of interferon-gamma release assay to detect latent tuberculosis infection in kidney transplant recipients. Transplant infectious disease : an official journal of the Transplantation Society.

$2017 ; 19(2)$. 
Figure 1. Countries of birth of transplant recipients

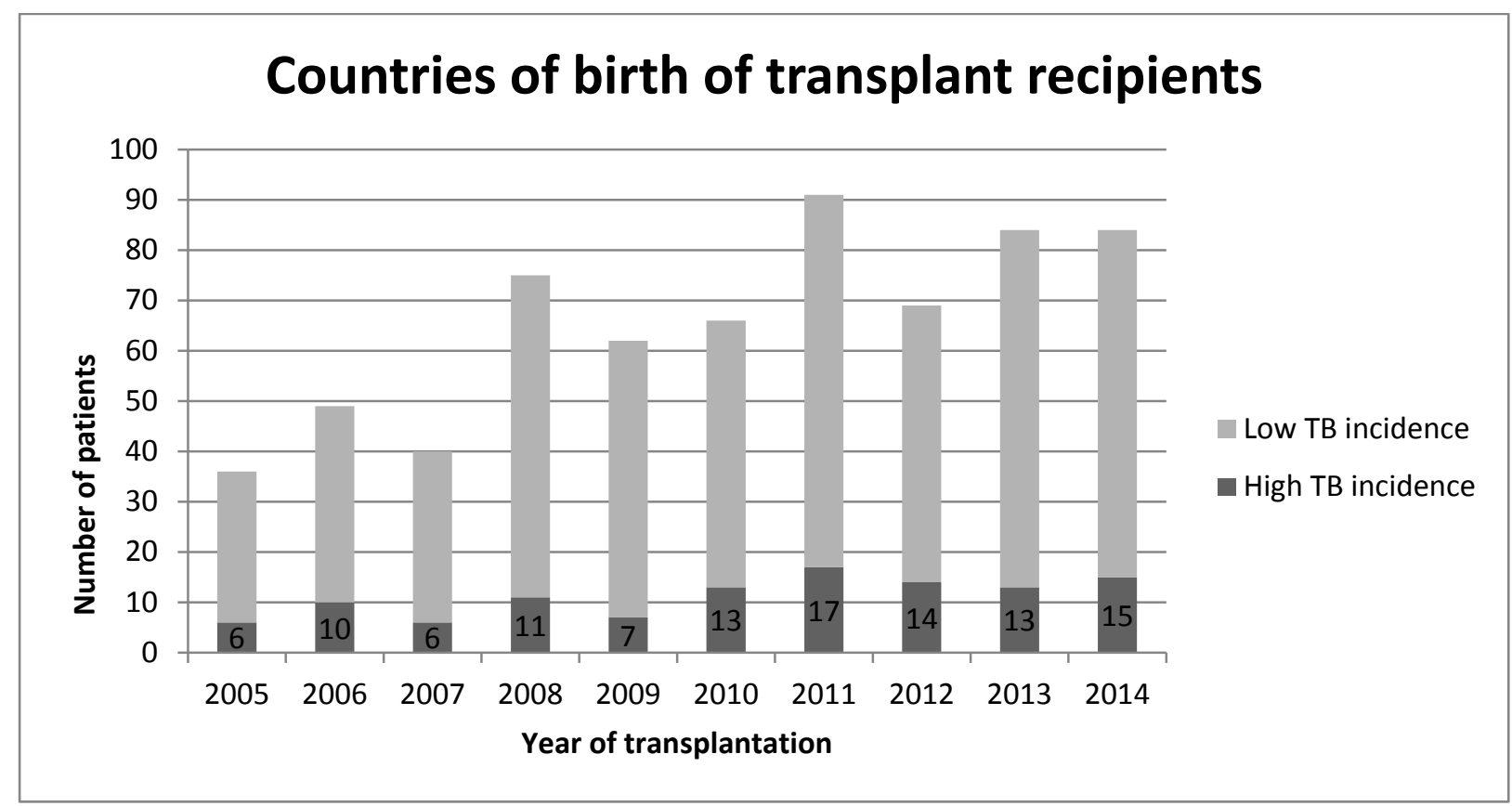

TB: tuberculosis.

This article is protected by copyright. All rights reserved. 
Figure 2. Screening and treatment cascade of IGRA testing performed pre-transplant

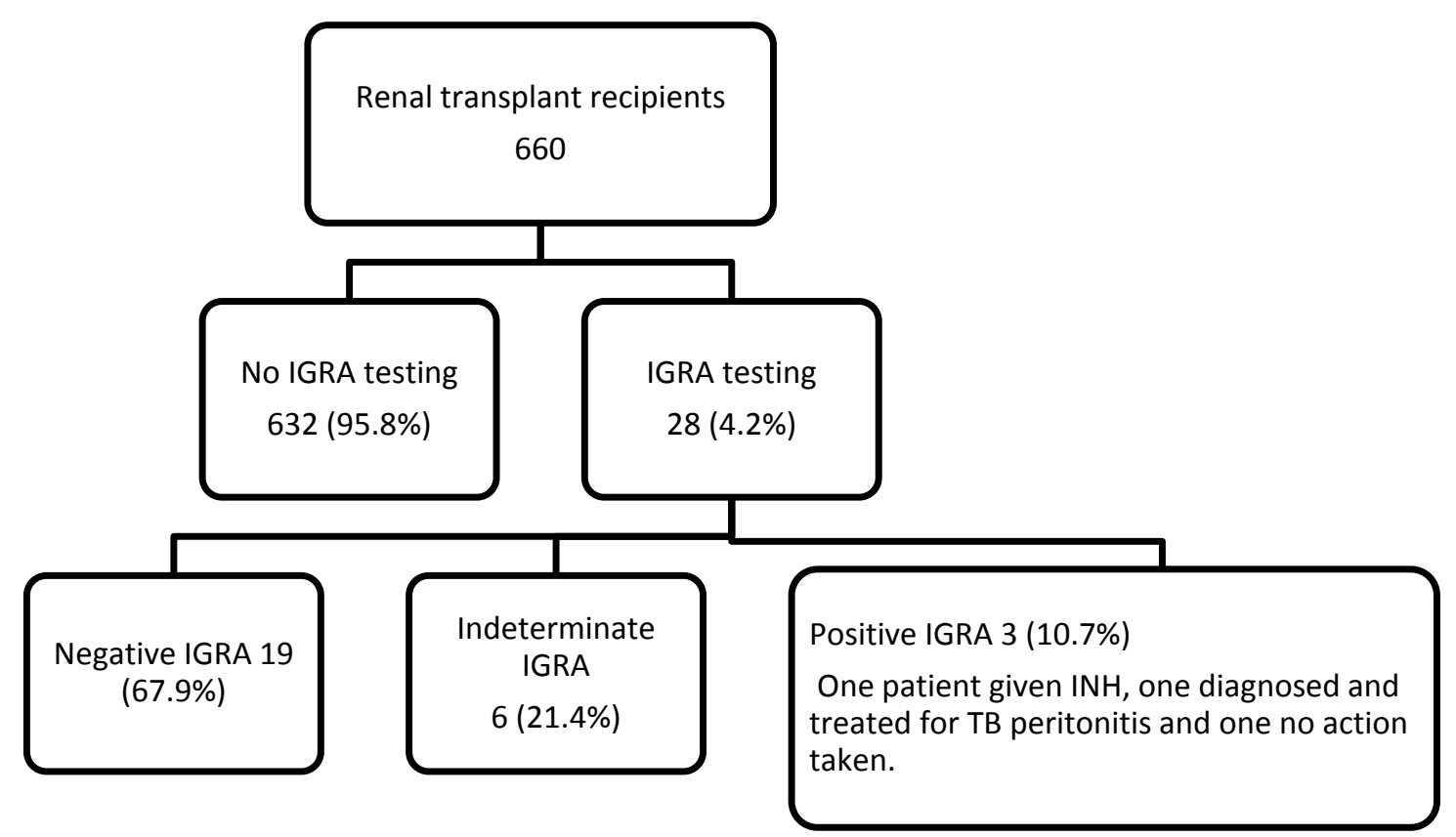

IGRA: Interferon release gamma release assay, INH: isoniazid, TB: tuberculosis 
Table 1. Characteristics of transplant recipients from 2005 to 2014.

\begin{tabular}{|c|c|c|c|c|}
\hline & $\begin{array}{l}\text { Entire cohort } \\
(n=660)\end{array}$ & $\begin{array}{l}\text { IGRA testing } \\
\text { performed }(n=89)\end{array}$ & $\begin{array}{l}\text { IGRA testing not } \\
\text { performed }(n=571)\end{array}$ & p-value \\
\hline $\begin{array}{l}\text { Age, median } \\
\text { (interquartile range) }\end{array}$ & $48.4(39-59)$ & $52(43-60)$ & $49(38-59)$ & 0.11 \\
\hline Male gender & $414(62.7 \%)$ & $60(67.4 \%)$ & $354(62.0 \%)$ & 0.29 \\
\hline \multicolumn{5}{|l|}{ Primary renal disease } \\
\hline Diabetes & $196(29.7 \%)$ & $16(18.0 \%)$ & $180(31.5 \%)$ & \multirow{6}{*}{0.016} \\
\hline Glomerulonephritis & $247(37.4 \%)$ & $39(43.8 \%)$ & $208(36.4 \%)$ & \\
\hline Reflux & $51(7.7 \%)$ & $13(14.6 \%)$ & $38(6.7 \%)$ & \\
\hline Vascular & $33(5.0 \%)$ & $5(5.6 \%)$ & $28(4.9 \%)$ & \\
\hline Cystic & $77(11.7 \%)$ & $7(7.9 \%)$ & $70(12.3 \%)$ & \\
\hline Other & $56(8.5 \%)$ & $9(10.1 \%)$ & $47(8.2 \%)$ & \\
\hline \multicolumn{5}{|c|}{ Dialysis prior to transplant } \\
\hline Pre-dialysis & $77(11.7 \%)$ & $8(9.0 \%)$ & $69(12.1 \%)$ & \multirow{3}{*}{0.716} \\
\hline Haemodialysis & $394(59.7 \%)$ & $56(62.9 \%)$ & $338(59.2 \%)$ & \\
\hline Peritoneal dialysis & $189(28.6 \%)$ & $25(28.1 \%)$ & $164(28.7 \%)$ & \\
\hline \multicolumn{5}{|l|}{ Type of transplant } \\
\hline Kidney-pancreas & $111(16.8 \%)$ & $6(6.7 \%)$ & $105(18.4 \%)$ & \multirow{3}{*}{0.015} \\
\hline Living donor & $194(29.4 \%)$ & $28(31.5 \%)$ & $166(29.1 \%)$ & \\
\hline Deceased donor & $355(53.8 \%)$ & $55(61.8 \%)$ & $300(52.5 \%)$ & \\
\hline \multicolumn{5}{|l|}{ Country of birth } \\
\hline High TB incidence & $102(15.5 \%)$ & $38(42.7 \%)$ & $64(11.3 \%)$ & 0.001 \\
\hline \multicolumn{5}{|l|}{ Comorbidities } \\
\hline Chronic lung disease & $43(6.5 \%)$ & $8(9.0 \%)$ & $35(6.1 \%)$ & 0.352 \\
\hline
\end{tabular}




\begin{tabular}{|l|c|c|c|c|}
\hline Coronary artery & $134(20.3 \%)$ & $19(21.3 \%)$ & $115(20.1 \%)$ & 0.778 \\
disease & & $8(9.0 \%)$ & $85(14.9 \%)$ & 0.188 \\
Peripheral vascular & $93(14.1 \%)$ & $1(1.1 \%)$ & $34(6.0 \%)$ & 0.072 \\
\hline Cerebrovascular & $35(5.3 \%)$ & & & 0.001 \\
\hline disease & $134(20.3 \%)$ & $7(7.9 \%)$ & $127(22.2 \%)$ & 0.092 \\
\hline Diabetes - type 1 & $92(13.9 \%)$ & $17(19.1 \%)$ & $75(13.1 \%)$ & \\
\hline
\end{tabular}

Data are number of individuals (proportion of cohort) except where indicated.

P-values where calculated using Fisher's exact test with a p-value of $<0.05$ considered significant. The Mann-Whitney test was used to calculate p-value for age.

IGRA: Interferon gamma release assay; TB: tuberculosis 
Table 2: Renal transplant patients with active tuberculosis infection

\begin{tabular}{|c|c|c|}
\hline Clinical scenario & $\begin{array}{l}\text { Site and Diagnosis of } \\
\text { TB }\end{array}$ & $\begin{array}{l}\text { Treatment and } \\
\text { Complications }\end{array}$ \\
\hline $\begin{array}{l}\text { 37F with failed renal } \\
\text { transplant who developed } \\
\text { fevers whilst on PD. }\end{array}$ & $\begin{array}{l}\text { Disseminated TB: } \\
\text { peritoneal, } \\
\text { intrathoracic lymph } \\
\text { nodes. TB culture } \\
\text { positive from } \\
\text { bronchial washings, } \\
\text { peritoneal fluid and } \\
\text { tissue. }\end{array}$ & $\begin{array}{l}\text { Diagnostic delay of } 4 \text { months - short period of } \\
\text { treatment with INH monotherapy for LTBI } \\
\text { which was complicated by visual changes. } \\
\text { Treatment complicated by haemolysis due to } \\
\text { moxifloxacin and significant nausea. } \\
\text { Completed } 6 \text { months of therapy. }\end{array}$ \\
\hline $\begin{array}{l}\text { 59M with cough and } \\
\text { fever } 15 \text { years after renal } \\
\text { transplantation. }\end{array}$ & $\begin{array}{l}\text { Pulmonary TB; initial } \\
\text { bronchoscopy culture } \\
\text { negative }\end{array}$ & $\begin{array}{l}\text { Long diagnostic delay ( } 9 \text { months) as initial } \\
\text { bronchoscopy negative for TB culture. } \\
\text { Eventual diagnosis made based on EBUS } \\
\text { guided biopsy. } \\
\text { Treatment complicated by rash. Died } 3 \text { months } \\
\text { into treatment of unknown cause (clinically } \\
\text { stable whilst on treatment). (unrelated to TB). }\end{array}$ \\
\hline $\begin{array}{l}\text { 54F with febrile illness } \\
\text { and night sweats } 10 \\
\text { months post renal } \\
\text { transplant. }\end{array}$ & $\begin{array}{l}\text { Disseminated TB - } \\
\text { pulmonary, pleural and } \\
\text { vertebral spine. } \\
\text { Diagnosis based on } \\
\text { PCR and culture (M. } \\
\text { orygis) from bronchial } \\
\text { washings }\end{array}$ & $\begin{array}{l}\text { Significant adverse reactions to rifampicin: } \\
\text { hyperbilirubinemia, fever, neutropenia and } \\
\text { hepatitis. Completed } 18 \text { months of therapy } \\
\text { (prolonged therapy as unable to tolerate } \\
\text { rifampicin) }\end{array}$ \\
\hline
\end{tabular}

Abbreviations: $\mathrm{F}=$ female; $\mathrm{M}=$ male; $\mathrm{TB}=$ tuberculosis; $\mathrm{INH}=$ isoniazid; $\mathrm{LTBI}=$ latent tuberculosis infection; $\mathrm{PD}=$ peritoneal dialysis; $\mathrm{EBUS}=$ endobronchial ultrasound 


\section{University Library}

\section{- M M N E R VA A gateway to Melbourne's research publications}

Minerva Access is the Institutional Repository of The University of Melbourne

Author/s:

Rafiei, N;Williams, J;Mulley, WR;Trauer, JM;Jenkin, GA;Rogers, BA

Title:

Mycobacterium tuberculosis: Active disease and latent infection in a renal transplant cohort

Date:

2019-05-01

Citation:

Rafiei, N., Williams, J., Mulley, W. R., Trauer, J. M., Jenkin, G. A. \& Rogers, B. A. (2019).

Mycobacterium tuberculosis: Active disease and latent infection in a renal transplant cohort. NEPHROLOGY, 24 (5), pp.569-574. https://doi.org/10.1111/nep.13386.

Persistent Link:

http://hdl.handle.net/11343/285755 